\title{
Recessive Epidermolysis Bullosa simplex- A case report
}

\author{
Yusuf SM, MBBS, MSC, FWACP; Uloko AE, BM BCh, FMCP
}

Department of Medicine, Aminu Kano Teaching Hospital, Kano, Nigeria

Correspondence: Dr Shehu Mohammed Yusuf; e-mail: shehumy@yahoo.com

\begin{abstract}
:
Background:Epidermolysis bullosa simplex (EBS) is characterized by intraepidermal blister formation, most commonly appearing in early infancy. Many variants of EBS exist; the four most common variants are inherited in an autosomal dominant fashion. The recessive forms are rare and less reported in our environment. The objective of this report is to describe the case of a young boy with a rare, recessive variant of EBS born to first-cousin parents.

Method: The case records of a twelve-year old Nigerian male being managed at the dermatology clinic of Aminu Kano Teaching Hospital (AKTH), Kano was reviewed. Relevant clinical photographs with informed consent and review of literature were carried out.

Results: A 12 year old Nigerian male, product of consanguineous marriage presented to the dermatology clinic with recurrent blistering of the skin since early childhood. The areas of the skin commonly affected include the knees, ankles, wrists and elbows with seasonal variations in the severity of lesions. His immediate younger brother is said to have similar skin lesions. Physical examination and histology were in keeping with EBS.

Conclusion: Recessive EBS though rare, does occur in our environment and should be suspected in males with blistering skin conditions that are products of consanguineous marriage.
\end{abstract}

Key words: Epidermolysis bullosa simplex, consanguinity, skin blistering

Introduction:

Inherited epidermolysis bullosa (EB) is a heterogeneous group of mechanobullous disorders characterized by painful blister formation as a result of minor trauma to the skin. It has been grouped into three major types according to the level of the blister formation: simplex, junctional, or dystrophic.1 EB simplex has been sub grouped based on gene/protein defects into several subtypes mostly inherited as autosomal dorminant, and only rarely autosomal recessive forms. 2

\section{Case report:}

A 12 year old male Nigerian, presented to the dermatology clinic of Aminu Kano Teaching Hospital (AKTH) with history of recurrent skin blisters. $\mathrm{He}$ is a product of a consanguineous marriage; being the third of seven siblings. The blisters affect the knees, ankles, wrists and elbows. These were first observed when he was 4-month old. Blisters were aggravated during summer and scarce during winter. The parents noticed significant improvement with fewer lesions appearing for shorter periods of time as the patient grew up. Healing of the blisters was accompanied by minimal scaring. There were initially no nail or hair changes. Over the last 5 years there has been sequential avulsion of his toe nails and blistering of the skin over the dorsum of the feet; this was attributed to frequent trauma sustained while playing football. Similar skin changes were also present on his immediate younger brother. The patient reported occurrence of similar lesions in two of his brothers. None of the parents or any member of the extended family had history of blistering. Physical examination revealed flaccid bullae over trauma prone sites such as the wrist, ankles, knees and elbows [Figure 1(A\&B)]. There were evidences of mild scaring of affected sites. The patient had avulsed toe nails (Figure 1c) but normal looking hairs and dentition. The mucosae of the mouth and eyes looked normal. A biopsy specimen of perilesional skin taken after slight mechanical provocation showed intraepidermal vesicles with very few inflammatory infiltrates (Figure 2). None availability of other diagnostic apparatus such as Transmission electron microscopy (EM), Immunofluorescence mapping (IFM) preclude further confirmatory diagnostic investigations. Despite the early onset of the disease, the absent family history of blistering, affectation of other siblings, consanguineous parental marital relationship and the clinical and histological findings were consistent with a diagnosis of recessive EBS.

Discussion:

EBS is commonly first seen at birth or in early infancy. It has an incidence of approximately 10-30 cases per million3-5. Several subtypes of EBS have been identified. Most cases result from mutations in cytokeratin genes 5 (KRT5) or 14 (KRT14). Epidermolysis bullosa simplex is most often expressed as an autosomal dominant trait but there have been a few reports of recessive EBS (REBS). These are also caused by mutations in the KRT5 or KRT14 genes.2 REBS

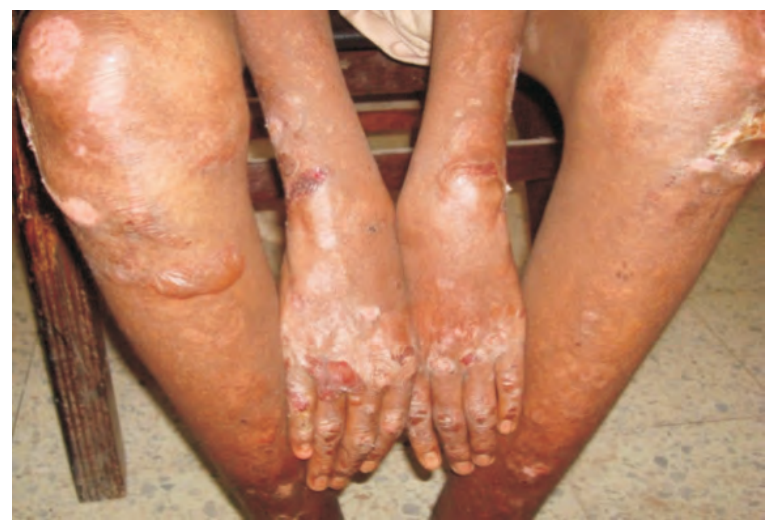

Figure 1:Bullae at trauma-prone sites with healed areas showing mild scaring 
also shows genotype-phenotype correlation between the location of the mutation within the keratin protein and the severity of the disorder. More cases of recessive EBS have proved to be "knock-outs" of K14, mostly showing severe generalised blistering due to complete ablation of K14 expression, with tonofilaments (keratin filament bundles) absent from basal keratinocytes on electron microscopy.6 A point mutation in the 1A domain of K14 outside the helix initiation motif produces a very mild Weber-Cockayne-like phenotype. This type of mutation has been rarely reported.7 It is likely that our patient's gene mutation most probably involves 1A domain of K14 due to the very mild nature of his condition. The absence of confirmatory tests made it impossible to give a molecular diagnosis.

Treatment for patients affected with EBS is in general supportive and preventive, consisting of avoidance of trauma, wound management, nutritional support and infection control. Genetic counselling is offered to couples affected with EBS.

\section{References:}

1. Fine, J. D., Eady, R. A., Bauer, E. A., et al. The classification of inherited epidermolysis bullosa (EB): Report of the Third International Consensus Meeting on Diagnosis and Classification of EB. J. Am.Acad. Dermatol. 2000;42:1051-1066

2. Horn HM, Tidman MJ. The clinical spectrum of epidermolysis bullosa simplex. Br J Dermatol 2000;142: 468-472,

3. Horn HM, Priestley GC, Eady RA, Tidman MJ. The prevalence of epidermolysis bullosa in Scotland. Br J Dermatol 1997;136: 560-564,

4. Inaba Y, Kitamura K, Ogawa Het al. A study on the estimation of prevalence of epidermolysis bullosa in Japan. Nippon Hifuka Gakkai Zasshi 1989;99:1021-1026,

5. McKenna KE, Walsh MY, Bingham EA. Epidermolysis bullosa in Northern Ireland. Br J Dermatol 127: 318-321, 1992.

6. Jonkman MF, Heeres K, Pas HH et al. Effects of keratin 14 ablation on the clinical and cellular phenotype in a kindred with recessive epidermolysis bullosa simplex. J Invest Dermatol 1996; 107:764-769.

Batta K, Rugg EL, Wilson NJ et al. A keratin 14 'knockout' mutation in recessive epidermolysis bullosa simplex resulting in less severe disease. Br J Dermatol 2000; 143: 691-696. 